\title{
ARTICLE
}

\section{Antiglucocorticoids in psychiatry ${ }^{\dagger}$}

\author{
Sean A. Mclsaac, Åsa Westrin \& Allan H. Young
}

Sean Mclsaac studied for his

Bachelor degree in biology at the University of British Columbia,

Vancouver, Canada, where he is currently a research assistant at the Institute of Mental Health. He worked for the Wellington Lab,

focusing on the interplay of lipid transporters in Alzheimer's disease. His present work, with Professor Allan Young, follows his interest in the impact of endocrine dysfunction in psychiatric disorders. Åsa

Westrin is a research psychiatrist in the Department of Clinical Neuroscience at Lund University, Sweden. Her main research interest involves stress-system alterations in depression and suicidal behaviour.

Allan Young currently holds the LEEF Endowed Chair in Research in the Department of Psychiatry at the University of British Columbia, Vancouver, Canada, where he is also the Director of the Institute of Mental Health. His research interests focus on the causes and treatments for severe psychiatric

illnesses, particularly mood

disorders.

Correspondence Professor Allan Young, Institute of Mental Health,

University of British Columbia, 5950 University Blvd, Vancouver, BC

V6T 2A1, Canada. Email: alyoung@ interchange.ubc.ca

tFor a commentary on this article see pp. 250-252, this issue.

\begin{abstract}
SUMMARY
Significant evidence has accrued suggesting that the hypothalamic-pituitary-adrenal (HPA) axis plays a role in some psychiatric disorders. This article reviews the physiology of the HPA axis, evidence of dysfunction in this axis in psychiatric illnesses and the role that this dysfunction might play in pharmacological treatment resistance. Future therapeutic strategies that may potentially arise from these researches are briefly outlined.
\end{abstract}

\section{DECLARATION OF INTEREST}

A.Y. holds related grants from the Stanley Medical Research Institute and the Medical Research Council (UK) and has filed a provisional patent concerning the use of antiglucocorticoids as an adjunctive to antidepressant therapies.

Many of the current drug treatments in psychiatry were discovered without any knowledge of the underlying 'disease processes' concerned. Theories of the mechanism of drug action were all developed post hoc and are predominantly concerned with monoamine neurotransmitter modulation. However, not all patients are treated successfully by these means and rates of full remission are frequently quite low. Current diagnostic categorisation of psychiatric disorders, such as unipolar depression, bipolar disorder and schizophrenia, is provisional and subject to ongoing revision. Ideally, drug development should be predicated on knowledge of 'drug targets' derived from understanding of the pathophysiological processes involved that are important for the illness in question. Although the central pathophysiological components of most psychiatric disorders remain unknown, a large body of evidence indicates that endocrine dysfunction, particularly of the hypothalamicpituitary-adrenal (HPA) axis, may be important for certain psychiatric illnesses, as indeed is the case for some physical illnesses. Factors related to the HPA axis may partially account for treatmentresistance (although, of course, a variety of factors contribute) and these may also suggest targets for development of new drugs with novel modes of action.

\section{The hypothalamic-pituitary-adrenal axis}

The HPA axis is an endocrine system comprising both central nervous system and peripheral endocrine tissue: the hypothalamus, pituitary and adrenal cortices are its major components. The HPA axis is regulated by external inputs from a number of brain regions, including the amygdala, hippocampus and nuclei within the midbrain. In addition, the HPA axis also contains a number of autoregulatory mechanisms. The paraventricular nucleus of the hypothalamus secretes the peptides corticotropin-releasing hormone $(\mathrm{CRH})$ and arginine vasopressin (AVP) into the microportal circulatory system of the pituitary stalk. These peptides have a synergistic effect on the release of adrenocorticotropic hormone (ACTH) from the anterior lobe of the pituitary. Cortisol, a glucocorticoid released from the adrenal cortex in response to ACTH, has a plethora of central and peripheral effects which are mediated primarily via glucocorticoid receptors of types I and II. Under normal homeostatic conditions, type I receptors are mostly saturated, owing to their high affinity for cortisol and other glucocorticoids, and type II glucocorticoid receptors are the more sensitive to stressor-dependant changes within the axis. It is by the glucocorticoid receptor that cortisol primarily exerts its negative feedback on the hippocampus, hypothalamus and pituitary. Under normal conditions, diurnal variation in cortisol levels is seen. In humans, this begins with markedly elevated serum concentrations shortly after awakening, trailing off to the lowest levels in the evening, presenting as a characteristic curve when concentrations are graphed over a $24 \mathrm{~h}$ period. Changes in glucocorticoid receptor number or function may be important in altering the homeostatic function of the HPA axis observed in healthy individuals. These regulatory mechanisms are important in determining basal levels and circadian fluctuations in cortisol levels (Dinan 2005).

\section{HPA abnormalities in psychiatric illnesses}

The first observations of abnormal cortisol levels in people with depression were made in England by Board and colleagues (Board 1957). Subsequent studies have shown that HPA hyperactivity, as variously manifested by hypersecretion of $\mathrm{CRH}$ and AVP, increased cortisol levels in plasma, urine, cerebrospinal fluid and saliva, exaggerated cortisol responses to ACTH, and enlarged hypothalamus, pituitary and adrenal glands, occurs in individuals with a number of severe mood disorders. 
In addition to unipolar depression, hypercortisolaemia has since been found in other psychiatric illnesses, including psychotic depression, bipolar disorder, schizophrenia and Alzheimer's disease (Nelson 1997; Walder 2000; Watson 2004; DeBattista 2005), although these abnormalities in cortisol levels have not always been found in milder depression (using ICD-10 criteria) (Cowen 2002). In severe depression, high doses of glucocorticoids have been shown to cause brief elevations in mood, suggesting that cortisol may actually be a resilience factor that the body releases to restore normal serotonergic function (Young AH 1994a). Hypersecretion of CRH and AVP, causing hypercortisolaemia, may be a result of impaired feedback mechanisms resulting from glucocorticoid receptor abnormalities, such as decreased receptor number or altered function (Dinan 2005). This view is supported by the demonstration of downregulated glucocorticoid receptor mRNA in post-mortem frontal and temporal cortices and hippocampi of individuals with unipolar or bipolar disorder or schizophrenia (Webster 2002).

The cortisol-suppressing activity of the synthetic glucocorticoid dexamethasone is useful as a measure of the functional integrity of the glucocorticoidreceptor-mediated negative feedback mechanism. Reports of cortisol non-suppression in response to dexamethasone in unipolar, bipolar and schizophrenic disorders suggest a primary glucocorticoid receptor abnormality in these disorders (Zhou 1987). Post-dexamethasone AVP levels have proven to be a sensitive measure of HPA dysfunction when compared with healthy controls, and levels are elevated in unipolar and bipolar disorder, in both currently symptomatic and remitted patients (Dinan 2005; Watson 2006).

(Key points 1.)

Supplementing the dexamethasone suppression test with subsequent administration of exogenous $\mathrm{CRH}$ is known as the dex/CRH test, and recent work utilising this technique has verified that HPA axis function is abnormal in bipolar disorder (Watson

\section{KEY POINTS 1}

- Robust evidence demonstrates abnormalities of the HPA axis in a number of psychiatric disorders

- Interaction between brain serotonergic systems and the HPA axis may be relevant to the pathogenesis of depressive symptoms and related cognitive deficits

- These depressive symptoms and cognitive deficits may be due to the neurocytotoxic effects of excessive cortisol
2004). This implicates the regulatory mechanisms that involve glucocorticoid receptors and AVP and $\mathrm{CRH}$ release in the HPA axis. Response to the dex/ $\mathrm{CRH}$ test has been found to resolve with successful treatment in acute unipolar depression (Kunugi 2006), but in bipolar disorder preliminary results show that the abnormality appears stable over time and is independent of mood state, persisting in euthymia (Watson 2004).

\section{Cortisol abnormalities in post-traumatic stress disorder}

Evidence is conflicting regarding the role of the HPA axis in post-traumatic stress disorder (PTSD). Some studies indicate dysfunction of the axis, showing hypocortisolaemia, rather than hypercortisolaemia, although elevated $\mathrm{CRH}$ is still reported (Yehuda 2001). The same group has found evidence indicating that ACTH is also elevated in PTSD; it is therefore likely that the dysfunction in the HPA axis lies in the output of the adrenal glands (Yehuda 2006). This would suggest that antiglucocorticoid treatments may not be able to be used in the same manner for PTSD (because of already low cortisol levels) as for disorders with hypercortisolaemia. Other research groups have found that salivary cortisol levels are actually normal in individuals with PTSD, and that hypercortisolaemia is found only in individuals with PTSD and comorbid major depressive disorder (Young EA 2004a).

\section{5-HT and HPA axis interaction}

Whether hypercortisolaemia is a contributing risk factor or a resulting effect of the disorders listed above has yet to be determined. However, when considering these putative pathophysiological processes it is important to note that there appears to be complex regulatory interaction between the HPA axis and brain serotonergic systems (for a review see Porter 2004).

Studies show that with successful treatment of depression using serotonergic antidepressants (i.e. selective serotonin reuptake inhibitors and serotonin and noradrenaline reuptake inhibitors - SSRIs and SNRIs), non-suppression of the dexamethasone suppression test and other HPA dysfunctions resolve, although this is not fully established for all other antidepressants (Harmer 2003). Precursors of serotonin (5-HT), including L-tryptophan and 5-hydroxytryptophan (5-HTP), have been shown to increase ACTH, $\mathrm{CRH}$ and cortisol levels. Some evidence suggests that 5-HT may even act directly on the adrenal glands to release cortisol (for reviews see Dinan 1996; Porter 2004). 
In vivo work on rats has shown that after a bilateral adrenalectomy, 5-HT synthesis is reduced in the hypothalamus, hippocampus and raphe nuclei (Dinan 1996). Further animal work on the somatodendritic 5-HT $\mathrm{HA}_{1 \mathrm{~A}}$ autoreceptor indicates that glucocorticoids modulate expression of these receptors in various regions of the brain (Man 2002). This has been replicated in normal human volunteers by using hydrocortisone to attenuate the effects of the partial serotonin agonist buspirone on the hypothermia and reduced duration of rapid eye movement sleep (Young AH 1994b).

The HPA overactivity observed in people with depression appears to alter the diurnal rhythms of the axis, eliminating the normal decrease in cortisol seen in the later part of the day and producing a more sustained release over time. One consequence of this flattened glucocorticoid rhythm may be a reduced clinical efficacy of antidepressant treatments such as chronic SSRIs. Gartside and collaborators modelled this flattened glucocorticoid rhythm in experimental animals and then examined the effects of this manipulation on the neuropharmacological effects of an SSRI (Gartside 2003). They used an implanted corticosteronereleasing pellet (compared with sham pellets) to reproduce the flattened glucocorticoid rhythm and then administered either fluoxetine or vehicle to rodents. In vivo microdialysis was then conducted to quantify effects on extracellular 5-HT levels. With fluoxetine treatment, 5-HT levels were significantly higher in the sham-pellet group than in the group whose glucocorticoid rhythm had been flattened by corticosterone-releasing pellets. This evidence suggests that elevated glucocorticoids (as found in mood disorders) reduce the ability of SSRIs to elevate extracellular brain 5-HT levels in the forebrain and thus impair the clinical efficacy of these drugs.

Similar findings were observed in a group of fluoxetine-resistant women, who demonstrated HPA-axis overactivity, compared with a group of matched women who were being treated successfully with fluoxetine (Young EA 2004b). Add-on treatment of an antiglucocorticoid drug remedied this non-response and demonstrated HPA axis interference with a conventional SSRI.

Interestingly, in people with remitted depression, the risk of relapse is greatly increased when cortisol levels are chronically increased (Zobel 2001). Thus, both lack of recovery and a higher risk of relapse may be related to increased cortisol levels, although this could be viewed as a manifestation of unresolved depression. The $5-\mathrm{HT}_{1 \mathrm{~A}}$ receptor number and function have been shown to be reduced in people with depression, and animal studies have shown that $5-\mathrm{HT}_{1 \mathrm{~A}}$ receptor binding is reduced after administration of corticosterone (Mendelson 1992). However, human studies attempting to replicate this possible regulatory mechanism have thus far shown mixed results (for a review see Porter 2004).

\section{Consequences of hypercortisolaemia}

It is widely recognised that a variety of medicinal steroids can cause neuropsychiatric adverse reactions. A number of case reports from the 1980s revealed that, though rare, even intranasal corticosteroid sprays can trigger relapse into episodes of both mania and psychosis after a period of sustained administration (Lewis 1983; Meyboom 1988; Goldstein 1989; Phelan 1989). Cushing's disease is an endocrine disorder in which hypercortisolaemia occurs, most commonly caused by a tumour in the pituitary but also occasionally by peripheral adrenal lesions. It is now established that cognitive impairments similar to those caused by medicinal steroids are seen across various conditions in which endogenous or exogenous corticosteroids are raised, including both Cushing's disease and severe mood disorders (Wolkowitz 1990).

Studies in experimental animals have shown deficits in learning and memory following chronic administration of glucocorticoids (Lupien 1997), as well as marked atrophy of neurons in the hippocampal formation. It has been postulated that a similar effect of cortisol may underlie some of the cognitive deficits observed in humans with severe mood disorders (Sapolsky 1986).

Clinical data suggest that cortisol treatment induces cognitive deficits in healthy humans, and these deficits appear to be attributed partly to the frontal lobe, suggesting that this brain area may also be sensitive to these effects of cortisol (Young AH 1999). The deficits in healthy volunteers are reversible, but this may not be entirely the case with the cognitive deficits induced by hypercortisolaemia associated with mood disorders if atrophy of tissue is irreversible in long-term illness (Young AH 1999; Thompson 2005). Indeed, duration of illness is known to be negatively correlated with severity of cognitive deficits (Cavanagh 2002). Therefore, early re-establishment of normal HPA activity in mood disorders may be an important therapeutic goal, before irremediable deficits in cognitive function can occur.

\section{Consequences of hypocortisolaemia}

Just as excess cortisol has detrimental consequences, so does a deficiency of glucocorticoids. Although elevated cortisol has neurotoxic effects on the above-mentioned regions, cortisol is also known to have a neuroprotective role and it is critical in 
regulating the innate immune responses of the central nervous system (Glezer 2004). In individuals with Addison's disease, primary adrenal failure results in hypocortisolaemia as one of the major presenting traits. Commonly, individuals report a decreased quality of life, due to chronic fatigue and a reduced sense of well-being, and although deficits in cognition are anecdotally acknowledged, they are not yet well-defined (Hunt 2000). These tentatively posited consequences of low cortisol levels are important to consider for putative antiglucocorticoid treatments in psychiatry, as therapy of this kind is likely to be most beneficial on a short-term basis.

\section{Suicide risk}

When looking at the influence of hypercortisolaemia on risk of suicide, it becomes important to differentiate between suicide attempt and completed suicide. In a number of post-mortem studies, findings have indicated increased measures of HPA axis activity in completed suicide (DoroviniZis 1987; Nemeroff 1988; Arato 1989; Lopez 1992; Raadsheer 1995). Similarly, non-suppression of cortisol has repeatedly been shown to be associated with subsequent completed suicide (Lester 1992). From this, one might hypothesise that treatment with antiglucocorticoids might have a specific influence on suicide risk.

This association between HPA axis hyperactivity and subsequent completed suicide seems to be most frequent among severely ill patients (Coryell 2006); it has been found in particular in inpatients with manifested suicide risk. Furthermore, among in-patients with a recent suicide attempt, an association has been demonstrated between non-suppression of cortisol and increased scores on the Suicide Assessment Scale (Westrin 2003). However, attempted suicide seems to be associated not with HPA axis hyperactivity (Lester 1992) but rather with hypoactivity of the HPA axis (Pfennig 2005). Consequently, when evaluating the effects of antiglucocorticoids in clinical practice and in future research, a thorough assessment of suicide risk, as well as suicidal behaviour, should be taken into consideration.

\section{Therapeutic targets}

There is increasing evidence to suggest that the consequences of HPA dysfunction described above are central to the pathogenesis of severe affective disorders and cognitive deficits (McQuade 2000). Modulation of the effects of hypercortisolaemia may provide potential treatments for mood disorders, and such strategies are the focus of considerable research interest.

\section{Dehydroepiandrosterone}

The adrenal steroid dehydroepiandrosterone (DHEA) has been used with some success in the treatment of depression (Wolkowitz 1999). The physiological functions of DHEA, and its sulphated conjugate DHEA-S, are numerous and it is now known to act as both an active hormone and a prohormone for sex steroids. It may have antidepressant qualities and it is speculated that its therapeutic effects may be accounted for by its potentially antiglucocorticoid properties (Wolkowitz 1999).

One proposed mechanism for the antiglucocorticoid effects of DHEA is that the molecule aids in reversing nuclear localisation of the glucocorticoid receptor caused by its interaction with cortisol (Cardounel 1999). Another explanation is that DHEA is partially metabolised to testosterone and oestrogen, both of which have effects on mood. Other work, in mice, shows that the hormone has dose-dependant neuroprotective effects in the hippocampus and suggests that this quality is attributed to the hormone directly, independently of its subsequent products (Cardounel 1999).

In a study of the molar ratio between cortisol and DHEA in drug-free patients with depression, we (A.Y. and colleagues) found reduced DHEA levels and a correspondingly elevated cortisol: DHEA ratio. This proved to be a more sensitive measure in depression than basal cortisol alone (Young AH 2002). It is well established now that age-related declines in DHEA levels possibly reduce the effectiveness of the body's 'natural' antiglucocorticoid mechanisms.

\section{Steroid synthesis inhibitors}

High cortisol levels can be lowered by inhibiting steroid synthesis from cholesterol. Ketoconazole, metyrapone and aminoglutethimide are three commonly employed compounds, each of which acts to inhibit multiple enzymes involved in the creation of cortisol from cholesterol. Early studies of their use as antidepressant therapies reported promising results, showing successful reduction of ACTH and cortisol levels, correlated with improvements in mood (Murphy 1997). Ketoconazole, when administered daily, reduced both cortisol levels and depressive symptoms within $72 \mathrm{~h}$ in an individual with treatmentresistant depression (Ravaris 1988). Another study examined whether the addition of metyrapone to standard serotonergic antidepressants induced a more rapid, efficacious and sustained treatment response in patients with major depression (Jahn 2004). Of 63 individuals with major depression 
and taking nefazodone or fluvoxamine, 33 were chosen at random also to receive metyrapone for 3 weeks of a 5 -week trial. Metyrapone was reported to be an effective adjunct, accelerating the onset of antidepressant action. Serum antidepressant levels did not differ between the groups, thus suggesting that this acceleration was not due to a peripheral pharmacokinetic effect. Both a better treatment outcome and a greater antidepressive effect, sustained for the period of observation, were noted in the group receiving metyrapone augmentation.

\section{Statins}

Cholesterol is the basic building block of steroid hormones, and although they do not act directly on hormones, cholesterol synthesis inhibitors ('statins') have been studied as potential means of reducing available adrenal steroids. It is of note that statins have been reported to be associated with an increased incidence of behavioural and personality disorders that may be hormonally influenced (Huffman 2007).

The use of atorvastatin and lovastatin has been linked to an increase in dopamine and homovanillic acid (HVA) levels (Ormiston 2004), but no significant effect has yet been shown on steroid hormones or serotonin. Moreover, lowering of cholesterol may actually be attributed to an increase in depressive symptoms. In a study of 20 patients with hypercholesterolaemia treated with a variety of drugs, cholesterol was effectively reduced whereas the number of depressive symptoms significantly increased, although remaining at subclinical levels in all participants (Delva 1996).

\section{Corticotropin-releasing hormone antagonists}

Oversecretion of CRH, resulting in hypercortisolaemia, may be normalised by acute blockade of $\mathrm{CRH}$ receptors in the pituitary by means of compounds such as antalarmin and astressin, the withdrawal of which causes an increased signal in the normal HPA feedback loop. These drugs are still in preclinical stages but preliminary results suggest that these $\mathrm{CRH}$ antagonists indeed have clinical potential (Broadbear 2004; French 2007). Other drug development programmes are targeting central CRH receptors that are further from the HPA axis. We await the results of ongoing clinical investigations.

\section{Glucocorticoid receptor agonists}

Activation of the glucocorticoid-receptor-mediated negative-feedback mechanism that regulates cortisol levels is another strategy for reducing circulating cortisol levels. The synthetic glucocorticoid dexamethasone given at doses of 3-4 mg for 4 days has putative antidepressant effects (Arana 1995). At this dose, dexamethasone does not enter the central nervous system and consequently central glucocorticoid receptors are not activated (Karssen 2005). However, glucocorticoid receptors at the level of the pituitary are activated, leading to a lowering of endogenous circulating cortisol. The brief course of dexamethasone administration in these studies avoids the side-effects associated with longer-term treatment.

\section{Glucocorticoid receptor antagonists}

Glucocorticoid receptor antagonists have also been advocated as agents with potential therapeutic properties for mood disorders. This is based on the ability of the glucocorticoid receptor antagonist to block any detrimental effect of hypercortisolaemia and on the theoretical ability of an antagonist to up-regulate its receptor. Administration of a glucocorticoid receptor antagonist results in an acute antiglucocorticoid effect, while presumably causing a compensatory up-regulation of glucocorticoid receptor numbers, leading to enhanced negative feedback on the HPA axis after the antagonist has been discontinued. Initial clinical studies using the antagonist RU-486 (mifepristone) have shown some positive results, but some clinical efficacy may have been masked by the prolonged administration of the drug (Murphy 1993). Animal studies suggest that glucocorticoid receptor numbers are increased rapidly (within hours) after the administration of RU-486, which may restore normal feedback, thus 'resetting' the HPA axis. Such data suggest that a brief period of treatment with the antagonist may be adequate for restoring normal HPA axis function.

RU-486 has been evaluated both as augmentation and as monotherapy in the treatment of psychotic major depression. In a group of in-patients with the disorder, an open-design, open-label study involving acute augmentation (7 days) of current medications with 600 or $1200 \mathrm{mg}$ RU-486 daily produced significant reductions in scores on the Brief Psychiatric Rating Scale and in measures of depressive symptoms compared with baseline, although patients who received only $50 \mathrm{mg}$ RU-486 daily showed little or no benefit (Belanoff 2002). Similar findings were replicated in a study in which a group of patients taking neither conventional antidepressant nor antipsychotic medications received 7 days of RU-486 treatment for psychotic major depression (DeBattista 2005).

One of us (A.Y.) has been involved in a doubleblind cross-over pilot study of RU-486 for treatmentresistant bipolar disorder (Young AH 2004). After baseline data were collected, the 20 participants received either placebo or $600 \mathrm{mg} /$ day RU-486 for 
7 days, followed by 14 days of wash-out, at which point the cross-over began. During the study, neurocognitive and neuroendocrine function and mood symptoms were measured weekly. Following treatment with RU-486, a selective improvement in neurocognitive functioning and significant improvements in mood symptoms were observed, compared with placebo. The improvement in cognition was inversely correlated with basal cortisol levels, adding to the plausibility that this was an antiglucocorticoid effect. These data require replication but provide preliminary evidence that glucocorticoid antagonists may be effective in the treatment of cognitive deficits and mood symptoms associated with bipolar disorder. Interestingly, a similar protocol in patients with schizophrenia suggested no beneficial effect on symptoms or cognitive impairments, possibly because these patients had little evidence of a dysfunctional HPA axis (Gallagher 2005).

(Key points 2.)

\section{Conclusions}

Our understanding of the biological origins of psychiatric illnesses is beginning to expand beyond the narrow realm of monoamine neurotransmitter function, and the past decade has seen an increased understanding of how the hypothalamic-pituitaryadrenal (HPA) axis is involved in a number of major disorders. One of the most common expressions of HPA axis dysfunction is an excess of the glucocorticoid cortisol. Hypercortisolaemia may impair the effectiveness of current medications and has been associated with a characteristic pattern of neurocognitive deficits. High levels of this hormone may also correlate with a greatly increased rate of suicide. Different medications have been used in clinical trials to counteract hyperactivity of the HPA axis and restore normality, and the effects on both depressive symptoms and neurocognition have been positive. Recent data suggest that glucocorticoid receptor agonists, antagonists and steroid synthesis inhibitors may be useful in the treatment of mood disorders (Gallagher 2008), although more definitive large-scale clinical trials will be required to fully establish efficacy of this treatment strategy.

\section{KEY POINTS 2}

The following may be useful in the treatment of mood disorders:

- glucocorticoid receptor agonists

- glucocorticoid receptor antagonists

- steroid synthesis inhibitors

\section{References}

Arana GW, Santos AB, Laraia MT, et al (1995) Dexamethasone for the treatment of depression: a randomized, placebo-controlled, double-blind trial. American Journal of Psychiatry; 152: 265-7.

Arato M, Banki CM, Bissette G, et al (1989) Elevated CSF CRF in suicide victims. Biological Psychiatry; 25: 355-9.

Belanoff JK, Rothschild AJ, Cassidy F, et al (2002) An open label trial of C-1073 (mifepristone) for psychotic major depression. Biological Psychiatry: 52: 386-92.

Board F, Wadeson R, Persky H (1957) Depressive affect and endocrine functions: blood levels of adrenal cortex and thyroid hormones in patients suffering from depressive reactions. Archives of Neurology and Psychiatry; 78: 612-20.

Broadbear JH, Winger G, Rivier JE, et al (2004) Corticotropin-releasing hormone antagonists, astressin B and antalarmin: differing profiles of activity in rhesus monkeys. Neuropsychopharmacology; 29: 1112-21.

Cardounel A, Regelson W, Kalimi M (1999) Dehydroepiandrosterone protects hippocampal neurons against neurotoxin-induced cell death: mechanism of action. Proceedings of the Society for Experimental Biology and Medicine; 222: 145-9.

Cavanagh JTO, Van Beck M, Muir W, et al (2002) Case-control study of neurocognitive function in euthymic patients with bipolar disorder: an association with mania. British Journal of Psychiatry; 180: 320-6.

Coryell W, Young E, Carroll B (2006) Hyperactivity of the hypothalamicpituitary-adrenal axis and mortality in major depressive disorder. Psychiatry Research; 142: 99-104.

Cowen PJ (2002) Cortisol, serotonin and depression: all stressed out? British Journal of Psychiatry; 180: 99-100.

DeBattista C, Belanoff J (2005) C-1073 (mifepristone) in the adjunctive treatment of Alzheimer's disease. Current Alzheimer Research; 2: $125-9$.

Delva NJ, Matthews DR, Cowen PJ (1996) Brain serotonin (5-HT) neuroendocrine function in patients taking cholesterol-lowering drugs. Biological Psychiatry, 39: 100-6.

Dinan TG (1996) Serotonin and the regulation of hypothalamic-pituitaryadrenal axis function. Life Sciences: 58. 1683-94.

Dinan TG, Scott LV (2005) Anatomy of melancholia: focus on hypothalamicpituitary-adrenal axis overactivity and the role of vasopressin. Journal of Anatomy: 207: 259-64.

Dorovini-Zis K, Zis AP (1987) Increased adrenal weight in victims of violent suicide. American Journal of Psychiatry, 144: 1214-5.

French JA, Fite JE, Jensen $\mathrm{H}$, et al (2007) Treatment with CRH-1 antagonist antalarmin reduces behavioral and endocrine responses to social stressors in marmosets (Callithrix kuhlii). American Journal of Primatology, 69: 877-89.

Gallagher P, Watson S, Smith MS, et al (2005) Effects of adjunctive mifepristone (RU-486) administration on neurocognitive function and symptoms in schizophrenia. Biological Psychiatry; 57: 155-61.

Gallagher P, Malik N, Newham J, et al (2008) Antiglucocorticoid treatments for mood disorders. Cochrane Database of Systematic Reviews; issue 1: CD005168.

Gartside SE, Leitch MM, Young AH (2003) Altered glucocorticoid rhythm attenuates the ability of a chronic SSRI to elevate forebrain 5-HT: implications for the treatment of depression. Neuropsychopharmacology; 28: $1572-8$.

Glezer, I, Rivest, S (2004) Glucocorticoids: protectors of the brain during innate immune responses. Neuroscientist, 10: 538-52

Goldstein ET, Preskorn SH (1989) Mania triggered by a steroid nasal spray in a patient with stable bipolar disorder. American Journal of Psychiatry: 146: $1076-7$

Harmer CJ, Bhagwagar Z, Shelley N, et al (2003) Contrasting effects of citalopram and reboxetine on waking salivary cortisol. Psychopharmacology, 167: 112-4. 


\begin{tabular}{|lllll}
\multicolumn{5}{l}{ MCO answers } \\
1 & 2 & 3 & 4 & 5 \\
af & af & af & at & af \\
bf & bf & bf & bf & bf \\
cf & ct & cf & cf & ct \\
df & df & df & df & df \\
et & ef & et & ef & ef
\end{tabular}

Huffman JC, Stern TA (2007) Neuropsychiatric consequences of cardiovascular medications. Dialogues in Clinical Neurosciences; 9 : 29-45.

Hunt PJ, Gurnell EM, Huppert FA, et al (2000) Improvement in mood and fatigue after dehydroepiandrosterone replacement in Addison's disease in a randomized, double blind trial. Journal of Clinical Endocrinology and Metabolism; 85: 4650-6.

Jahn H, Schick M, Kiefer F, et al (2004) Metyrapone as additive treatment in major depression: a double-blind and placebo-controlled trial. Archives of General Psychiatry, 61: 1235-44.

Karssen AM, Meijer OC, Berry A, et al (2005) Low doses of dexamethasone can produce a hypocorticosteroid state in the brain. Endocrinology, 146: 5587-95.

Kunugi $\mathrm{H}$, Ida I, Owashi T, et al (2006) Assessment of the dexamethasone/ $\mathrm{CRH}$ test as a state-dependent marker for hypothalamic-pituitary-adrenal (HPA) axis abnormalities in major depressive episode: a multicenter study. Neuropsychopharmacology, 31: 212-20.

Lester D (1992) The dexamethasone suppression test as an indicator of suicide: a meta-analysis. Pharmacopsychiatry, 25: 265-70.

Lewis LD, Cochrane GM (1983) Psychosis in a child inhaling budesonide. Lancet, 2 (8350): 634

Lopez JF, Palkovits M, Arato M, et al (1992) Localization and quantification of pro-opiomelanocortin mRNA and glucocorticoid receptor mRNA in pituitaries of suicide victims. Neuroendocrinology, 56: 491-501.

Lupien SJ, McEwen BS (1997) The acute effects of corticosteroids on cognition: integration of animal and human model studies. Brain Research Reviews; 24: 1-27.

Man MS, Young AH, McAllister-Williams RH (2002) Corticosterone modulation of somatodendritic 5-HT1A receptor function in mice. Journal of Psychopharmacology, 16: 245-52.

McQuade R, Young AH (2000) Future therapeutic targets in mood disorders: the glucocorticoid receptor. British Journal of Psychiatry, 177: 390-5.

Mendelson SD, McEwen BS (1992) Quantitative autoradiographic analyses of the time course and reversibility of corticosterone-induced decreases in binding at 5-HT1A receptors in rat forebrain. Neuroendocrinology, 56 : 881-8.

Meyboom RH, de Graaf-Breederveld N (1988) Budesonide and psychic side effects. Annals of Internal Medicine; 109: 683.

Murphy BE, Filipini D, Ghadirian AM (1993) Possible use of glucocorticoid receptor antagonists in the treatment of major depression: preliminary results using RU 486. Journal of Psychiatry and Neuroscience; 18: 20913

Murphy BE (1997) Antiglucocorticoid therapies in major depression: a review. Psychoneuroendocrinology, 22 (suppl 1): s125-32.

Nelson JC, Davis JM (1997) DST studies in psychotic depression: a metaanalysis. American Journal of Psychiatry, 154: 1497-503.

Nemeroff CB, Owens MJ, Bissette G, et al (1988) Reduced corticotropin releasing factor binding sites in the frontal cortex of suicide victims. Archives of General Psychiatry, 45: 577-9.

Ormiston T, Wolkowitz OM, Reus VI, et al (2004) Hormonal changes with cholesterol reduction: a double-blind pilot study. Journal of Clinical Pharmacy and Therapeutics; 29: 71-3.

Pfennig A, Kunzel HE, Kern N, et al (2005) Hypothalamus-pituitaryadrenal system regulation and suicidal behavior in depression. Biological Psychiatry, 57: 336-42.

Phelan MC (1989) Beclomethasone mania. British Journal of Psychiatry, 155: 871-2.

Porter RJ, Gallagher P, Watson S, et al (2004) Corticosteroidserotonin interactions in depression: a review of the human evidence. Psychopharmacology, 173: 1-17

Raadsheer FC, van Heerikhuize JJ, Lucassen PJ, et al (1995) Corticotropinreleasing hormone mRNA levels in the paraventricular nucleus of patients with Alzheimer's disease and depression. American Journal of Psychiatry, 152: $1372-6$.
Ravaris CL, Sateia MJ, Beroza KW, et al (1988) Effect of ketoconazole on a hypophysectomized, hypercortisolemic, psychotically depressed woman. Archives of General Psychiatry, 45: 966-7.

Sapolsky RM, Krey LC, McEwen BS (1986) The neuroendocrinology of stress and aging: the glucocorticoid cascade hypothesis. Endocrine Reviews; 7: 284-301.

Thompson JM, Gallagher P, Hughes JH, et al (2005) Neurocognitive impairment in euthymic patients with bipolar affective disorder. British Journal of Psychiatry, 186: 32-40.

Walder DJ, Walker EF, Lewine RJ (2000) Cognitive functioning, cortisol release, and symptom severity in patients with schizophrenia. Biological Psychiatry, 48: 1121-32.

Watson S, Gallagher P, Ritchie JC, et al (2004) Hypothalamic-pituitaryadrenal axis function in patients with bipolar disorder. British Journal of Psychiatry, 184: 496-502

Watson S, Gallagher P, Ferrier IN, et al (2006) Post-dexamethasone arginine vasopressin levels in patients with severe mood disorders. Journal of Psychiatric Research; 40: 353-9.

Webster MJ, Knable MB, O'Grady J, et al (2002) Regional specificity of brain glucocorticoid receptor mRNA alterations in subjects with schizophrenia and mood disorders. Molecular Psychiatry, 7: 985-94.

Westrin A, Nimeus A (2003) The dexamethasone suppression test and CSF-5-HIAA in relation to suicidality and depression in suicide attempters. European Psychiatry, 18: 166-71.

Wolkowitz OM, Reus VI, Weingartner H, et al (1990) Cognitive effects of corticosteroids. American Journal of Psychiatry, 147: 1297-303.

Wolkowitz OM, Reus VI, Keebler A, et al (1999) Double-blind treatment of major depression with dehydroepiandrosterone. American Journal of Psychiatry, 156: 646-9

Yehuda, R (2001) Biology of posttraumatic stress disorder. Journal of Clinical Psychiatry, 62 (suppl 17): 41-6.

Yehuda $\mathrm{R}$ (2006) Advances in understanding neuroendocrine alterations in PTSD and their therapeutic implications. Annals of the New York Academy of Sciences; 1071: 137-66.

Young AH (1994a) Glucocorticoids, serotonin and mood. British Journal of Psychiatry, 165: 271-2.

Young AH, Sharpley AL, Campling GM, et al (1994b) Effects of hydrocortisone on brain 5-HT function and sleep. Journal of Affective Disorders: 32: $139-46$.

Young AH, Sahakian BJ, Robbins TW, et al (1999) The effects of chronic administration of hydrocortisone on cognitive function in normal male volunteers. Psychopharmacology, 145: 260-66.

Young AH, Gallagher P, Porter RJ (2002) Elevation of the cortisoldehydroepiandrosterone ratio in drug-free depressed patients. American Journal of Psychiatry, 159: 1237-9.

Young AH, Gallagher P, Watson S, et al (2004) Improvements in neurocognitive function and mood following adjunctive treatment with mifepristone (RU-486) in bipolar disorder. Neuropsychopharmacology, 29: $1538-45$

Young EA, Breslau N (2004a) Saliva cortisol in posttraumatic stress disorder: a community epidemiologic study. Biological Psychiatry, 56: 205-9.

Young EA, Altemus M, Lopez JF, et al (2004b) HPA axis activation in major depression and response to fluoxetine: a pilot study. Psychoneuroendocrinology, 29: 1198-204.

Zhou, DF, Shen, YC, Shu, LN, et al (1987) Dexamethasone suppression test and urinary MHPG X SO4 determination in depressive disorders. Biological psychiatry, 22: 883-91.

Zobel AW, Nickel T, Sonntag A, et al (2001) Cortisol response in the combined dexamethasone/CRH test as predictor of relapse in patients with remitted depression: a prospective study. Journal of Psychiatric Research; 35: 83-94. 
$\mathrm{MCOs}$

1 Cortisol is responsible for negative feedback in the HPA axis, and mainly by acting on:

a the hippocampus alone

b the hypothalamus alone

c the pituitary alone

d the hippocampus and the pituitary

e the hippocampus, the hypothalamus and the pituitary.

2 Abnormalities of the HPA axis that may be common in mood disorders include:

a glucocorticoid receptor function

b increased AVP levels

c decreased CRH levels d volume of the pituitary gland

e adrenal sensitivity to ACTH.

3 Clinical trials suggest that symptoms of depression may be improved by:
a $\mathrm{CRH}$ agonists
b antalarmin
c astressin
d atorvastatin
e RU-486.

4 Glucocorticoid receptor antagonists are thought to exert their therapeutic effect by:

a up-regulating glucocorticoid receptor receptors

b down-regulating glucocorticoid receptor receptors

c blocking conversion of DHEA to cortisol

$d$ blocking the effects of ACTH

e blocking the effects of CRH and AVP.

5 A factor increasing risk for dying by suicide may be:

a female gender

b hypocortisolaemia

c hypercortisolaemia

d dexamethasone hypersensitivity

e age between $25-35$ years 